score group, $12 \geqq$ : low score group), AUDIT score (0-14: group I, 15-19: group II, 20-: group III), job status (managerial or not), overtime work (per month, $<40$ hours: mild, 40-80 hours: middle, $>80$ hours: severe), quantitative overload (high/middle/low), qualitative overload (high/middle/low), stress with interpersonal relationships (high/middle/low), and job control (high/middle/low), as independent variables.

Results Depression tended to be higher in group II of AUDIT (OR: $1.40,95 \% \mathrm{CI}: 0.99$ to $1.96, \mathrm{p}=0.54$ ) and group III (OR: 2.45 , 95\% CI: 1.36 to $4.42, \mathrm{p}=0.003$ ). About overtimework, the severe group was more depressive than mild group (OR: $2.38,95 \% \mathrm{CI}: 1.45$ to $3.90, \mathrm{p}<0.001)$. More qualitative overload caused stronger depression (OR: 2.45, 95\% CI: 1.43 to $4.22, \mathrm{p}<0.001)$. Stress with interpersonal relationships also was related to depression (OR: 9.49, 95\% CI: 4.94 to 18.22, $\mathrm{p}<0.001)$.

Conclusion Higher AUDIT score seemed to show higher risk of developing depression. The wrong way of using alcohol may be an exacerbating factor of depression. We should be careful to drinking on the purpose of releasing stress.

\section{ASSOCIATION BETWEEN JOB STRESS AND OCCUPATIONAL INJURIES AMONG KOREAN FIREFIGHTERS: A NATIONWIDE CROSS-SECTIONAL STUDY}

\begin{abstract}
${ }^{1,2}$ Yeong-Kwang Kim, ${ }^{3}$ Yeon-Soon Ahn, ${ }^{4}$ Kyoo-Sang Kim, ${ }^{1,2,5,6} \mathrm{Jin}-\mathrm{Ha}$ Yoon, 1,2,5,6Jaehoon Roh*. 'Graduate School of Public Health, Yonsei University, South Korea, Seoul; ${ }^{2}$ The Institute for Occupational Health, Yonsei University College of Medicine, South Korea, Seoul; ${ }^{3}$ Department of Occupational and Environmental Medicine, Dongguk University Ilsan Hospital, South Korea, Ilsan; ${ }^{4}$ Department of Occupational and Environmental Medicine, Seoul Medical Centre, South Korea, Seoul; ${ }^{5}$ Department of Preventive Medicine, Yonsei University College of Medicine, South Korea, Seoul; ${ }^{6}$ Incheon Workers' Health Centre, South Korea, Incheon
\end{abstract}

\subsection{6/oemed-2018-ICOHabstracts. 1052}

Introduction Firefighters are responsible for the safety of citizens, and perform functions that include fire suppression and emergency medical services (EMS). As such, they are exposed to physical or chemical hazards that lead to high rates of occupational injuries. Despite a plethora of studies, there have been only a few systematic investigations to identify factors influencing occupational injuries among firefighters. In this study, which is based on a survey of all Korean firefighters, we aimed to investigate the existence of a correlation between job stress and occupational injury among firefighters.

Methods Cross-sectional study was conducted via a survey targeting firefighters in South Korea between July and November 2007. A questionnaire was mailed to 30630 firefighters; $25615(83.6 \%)$ responded. Statistical analysis was performed using the SAS 9.2 software (SAS Institute Inc., Cary, NC, USA).

Results Among fire suppression personnel, high job demands $(\mathrm{OR}=1.49,95 \% \mathrm{CI}: 1.25$ to 1.77$)$, high interpersonal conflicts $(\mathrm{OR}=1.18,95 \% \mathrm{CI}: 1.02$ to 1.37$)$, a poor organisational system $(\mathrm{OR}=1.33$, 95\% CI: 1.14 to 1.55$)$, and a negative workplace environment $(\mathrm{OR}=1.41,95 \% \mathrm{CI}: 1.21$ to 1.64$)$, and high job demands $(\mathrm{OR}=1.22,95 \% \mathrm{CI}$ : 1.01 to 1.47$)$ were associated with the occurrence of occupational injury. Among emergency medical services personnel, high job demands $(\mathrm{OR}=1.26,95 \% \mathrm{CI}: 1.03$ to 1.54$)$, high interpersonal conflicts $(\mathrm{OR}=1.40,95 \% \mathrm{CI}: 1.19$ to 1.66$)$, a poor organisational system $(\mathrm{OR}=1.55,95 \% \mathrm{CI}: 1.30$ to 1.85$)$, lack of reward $(\mathrm{OR}=1.43,95 \% \mathrm{CI}: 1.21$ to 1.69$)$ and a negative workplace environment $(\mathrm{OR}=1.30,95 \% \mathrm{CI}: 1.10$ to 1.54$)$ were associated with the occurrence of occupational injury.

Conclusion High job stress among firefighters was associated with both the occurrence of occupational injury, and also with an increased frequency of injuries. Therefore, job stress should be addressed to prevent occupational injuries among firefighters.

\section{DIFFERENCES BETWEEN PERITONEAL AND PLEURAL MESOTHELIOMA IN LOMBARDY, ITALY}

${ }^{1} \mathrm{D}$ Consonni*, ${ }^{2} \mathrm{M}$ Mendola, ${ }^{1} \mathrm{~B}$ Dallari, ${ }^{2} \mathrm{M}$ Sokooti, ${ }^{3} \mathrm{R}$ Tabibi, ${ }^{2} \mathrm{R}$ Callegari, ${ }^{4} \mathrm{~L}$ Riboldi, ${ }^{1} \mathrm{C}$ Mensi. 'Epidemiology Unit, Fondazione IRCCS Ca' Granda Ospedale Maggiore Policlinico, Milan, Italy, ${ }^{2}$ Università degli Studi di Milano, Milan, Italy; ${ }^{3}$ Abadan School of Medical Sciences, Abadan, Iran; ${ }^{4}$ Workers Health Protection and Promotion Unit, Fondazione IRCCS Ca' Granda Ospedale Maggiore Policlinico, Milan, Italy

\subsection{6/oemed-2018-ICOHabstracts. 1053}

Introduction Asbestos is the main risk factor for development of both peritoneal (PEM) and pleural (PLM) mesothelioma, but characteristics of this two diseases and their association with asbestos show some differences. We examined characteristics of PEM and PLM in Lombardy, Italy, the most populated (currently, 10 million residents) and industrialised Italian region.

Methods The RML, established in 2000, is a population-based registry which records all cases of mesothelioma among Lombardy residents. It is part of a national network of mesothelioma registries (ReNaM). Notification of mesothelioma cases is compulsory by law. Additional sources of mesothelioma cases include hospital admission, mortality, occupational diseases, and pathology databases. Verified mesothelioma cases are interviewed about past asbestos exposure using a standardised questionnaire. For this study, from the RML database we selected PEM and PLM cases diagnosed in 2000-2014. We investigated asbestos exposure, presence of asbestosis or pleural plaques, and past employment in economical sectors.

Results We identified 300 PEM and 5,011 PLM cases. Incidence rates (per 1.000.000 person-years, world standardised) of PEM were 2.4 (men) and 1.7 (women), compared with 52.4 and 19.9 for PLM. Asbestosis (both genders) and pleural plaques (men) were more frequent among PEM cases. Occupational asbestos exposure was similar in PEM and PLM cases. We found higher proportions of PEM employed in the asbestos cement production.

Conclusion The higher frequency of pleural plaques in PEM cases and the similar frequency of past occupational asbestos exposure confirm the association between asbestos exposure and peritoneal mesothelioma. The higher proportions of asbestosis and of past employment in the asbestos-cement sector among PEM cases suggest a possible role of high exposures to asbestos in the peritoneal mesothelioma genesis. 